\title{
Applying the fuzzy ART algorithm to distribution network design
}

\author{
Mazaher Ghorbani $^{\mathrm{a}^{*}}$, Reza Tavakkoli-Moghaddam ${ }^{\mathrm{b}}$ Jafar Razmi $^{\mathrm{b}}$ and S. Mohammad Arabzad
}

${ }^{a}$ Department of Industrial Engineering, Najafabad Branch, Islamic Azad University, Isfahan, Iran

${ }^{b}$ Department of Industrial Engineering, College of Engineering, University of Tehran, Tehran, Iran

\begin{tabular}{l}
\hline A R T I C L E I N F O \\
\hline Article history: \\
Received July 15, 2011 \\
Received in Revised form \\
September, 12, 2011 \\
Accepted 28 September 2011 \\
Available online \\
3 October 2011 \\
\hline Keywords: \\
Distribution network \\
Fuzzy ART \\
Categorizing \\
Partner selection
\end{tabular}
A B S T R A C T

\begin{abstract}
Distribution network design is an important issue in supply chain management and plays an important role in making new market development. Because of JIT philosophy, most of managers now have focused on designing appropriate distribution networks. Thus, categorizing distributors and selecting the best ones are crucial for companies. This paper provides a new method to categorize and select distributors. The fuzzy Adaptive Resonance Theory (ART) algorithm is utilized to categorize distributors according to their similarity. To improve the performance of the algorithm, we train the algorithm using the past data. Finally, a numerical example is illustrated to examine the validity of the proposed algorithm.
\end{abstract}

Partner selection

(C) 2012 Growing Science Ltd. All rights reserved.

\section{Introduction}

Supply chain management is the coordination of production, inventory, location and transportation among the participants in a supply chain to achieve the best combination of responsiveness and efficiency for the market being served (Hugos, 2006). One of the important areas in supply chain management (SCM) is partner selection. The competitive advantage of SCM is not only to focus on its core business, but also to establish long-term cooperative partnership with partners. Selection problems are very important in many real-life decision situations. Mousavi et al. (2011a) proposed a fuzzy stochastic approach for multi-attribute group decision making in uncertain situations. They illustrated the effectiveness of their model by applying it on a risk selection problem.

Mousavi et al. (2011b) also proposed an integrated DELPHI-AHP-PROMETHEE methodology for a plant location selection problem. In the area of supplier selection, numerous studies are found. Many researchers have investigated the importance of supplier selection problems and their key roles in achieving SCM goals (Bhattacharya et al., 2010; Punniyamoorthy et al., 2011; Vanteddu et al., 2010). A number of methodologies are applied in practice, such as linear and non-linear programing, mixed-

\footnotetext{
* Corresponding author. Tel: +989111537330

E-mail addresses: mazaher.ghorbani@gmail.com (M. Ghorbani) 
integer programming, multi-objective linear programming (Fassnacht \& Koese, 2006; Narasimhan et al., 2006; Talluri \& Narasimhan, 2005). Along these traditional methods, a number of studies have applied the fuzzy theory to supplier selection problems (Razmi et al., 2009; Wang et al., 2009).

Although numerous studies have been done in the context of supplier selection problems, there are a few studies in the context of distributor selection problems. Zou et al. (2011) introduced a rough setbased approach to distributor selection in a supply chain. They proposed a methodology, which is able to perform rule induction for distributors. Lin and Chen (2008) stated that there is little empirical research investigating manufacturers' selection of distributors and then tried to move researchers toward this area by proposing important factors when selecting distributors. Wang and Kess (2006) investigated the distributor selection problem by a case study. They mentioned that task and partnerrelated dimensions in partner selection of international joint ventures that were useful in the distributor relationship. Sharma et al. (2004) proposed a composite distributor performance index (DPI) to evaluate the distributors' performance.

\subsection{Overview of adaptive resonance theory (ART)}

The ART network is a neural algorithm in order to cluster arbitrary data into groups with similar features (Pacella et al., 2004). This network consists of input and output layers. The input layer takes a set of input vectors and gives clusters as output. Input vectors, which are close to each other according to a specific similarity measure, are mapped to the same cluster. If the input does not match any of the stored patterns, the new category can be existed. The ART has the orienting and attention subsystems. These subsystems are responsible for categorization and whether to accept it or not, respectively. Table 1 shows the classical ART clustering algorithms.

Table 1

ART models

\begin{tabular}{ll}
\hline ART algorithms & Description \\
\hline ART1 & Can stably learn to categorize binary inputs. \\
ART2 & Can learn to categorize analog patterns presented in an arbitrary order. \\
ART2A & $\begin{array}{l}\text { This algorithm is the fast version of ART2. } \\
\text { ART3 }\end{array}$ \\
$\begin{array}{l}\text { Extends ART by incorporating 'chemical transmitters' to control the search process in a } \\
\text { hierarchical ART structure. }\end{array}$ \\
$\begin{array}{ll}\text { Can rapidly self-organize stable categorical mappings between } M \text {-dimensional input vectors and } \\
N \text {-dimensional output vectors. }\end{array}$ \\
\hline Fuzzy ART & Incorporating computations from fuzzy set theory into ART1. \\
\hline
\end{tabular}

\subsubsection{Fuzzy ART}

The fuzzy ART neural network was first introduced by Carpenter et al. in 1991 (Aydin Keskin et al., 2006; Lopes et al., 2005). The fuzzy ART is an unsupervised learning algorithm, which is capable of learning in both off-line and on-line training modes. It is the most recent adaptive resonance framework, which provides a unified architecture for both binary and continuous value inputs. The generalization of learning both analog and binary input patterns is achieved by replacing the appearance of the logical AND intersection operator $(\cap)$ in ART1 by the MIN operator $(\wedge)$ of the fuzzy set theory (Pacella et al., 2004). According to Aydin Keskin et al. (2006), the fuzzy ART involves three main differences in comparison with ART1:

- There is a single weight vector connection.

- Non-binary inputs can be processed.

- In addition to vigilance threshold $(\rho)$, choice parameter $(\alpha)$ and learning rate $(\beta)$ should be determined.

Reviewing the literature of the fuzzy ART shows that in addition to its simplicity, this algorithm has been used frequently by researchers in recent years (Aydın Keskin et al., 2006; Aydın Keskin \& Ozkan, 2009; Pandian \& Mahapatra, 2009; Pacella \& Semeraro, 2011). Fig. 1 shows the basic fuzzy ART architecture. 


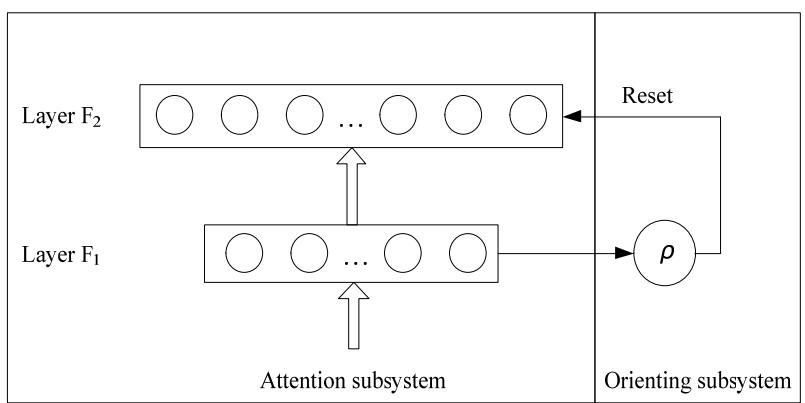

Fig. 1. Topological structure of the fuzzy ART

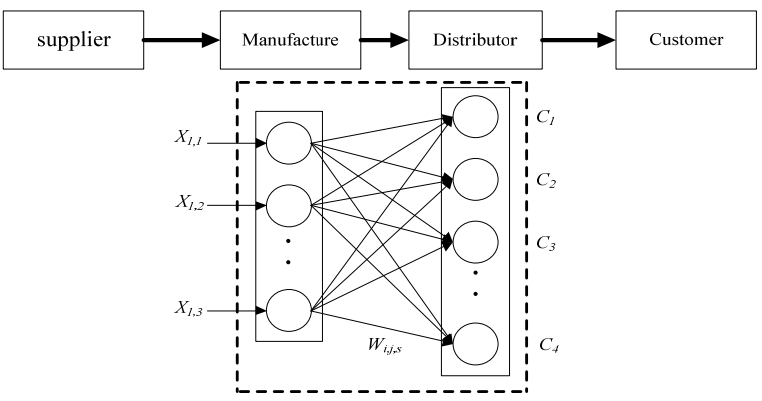

Fig. 2. Conceptual model of a supply chain with the fuzzy ART model for distributor selection

This paper applies the fuzzy ART's classification ability to the distributor categorization and selection area. The fuzzy ART methodology is able to categorize the candidate distributors according to the similarity between input values. Furthermore, some modifications are applied to enhance the classification ability of the algorithm. First, complement coding is used for the normalized data, and then the neural network is trained. The remainder of this paper is organized as follows. The basic concepts, definitions and notations of the proposed algorithm for distributor categorization and selection are introduced in Section 2. In Section 3, an illustrative numerical example is presented, after which this study discusses and shows how the proposed method is effective. Finally, conclusions are presented in Section 4.

\section{Fuzzy ART for distribution network design}

A distributor is a firm, which takes ownership of important inventories of products, in which distributor buy from producers and sell to consumers. For the customer, distributors fulfill the 'Time and Place' function, in which they deliver products when and where the customer wants them (Kuo \& Liang, 2011). The distributor in a supply chain is not only an important link connecting manufacturing and final customers to transfer products and value, but also the first line listening to customers' voice to directly grasp the pulse of demand. In this paper, the fuzzy ART-based algorithm is proposed for distributor categorization and selection. Fig. 2 shows the phases of the proposed algorithm and the applied fuzzy ART model for distributor selection, respectively. In the following, the stepwise explanation of the proposed method is discussed.

Phase 1. Determining team members and evaluation prerequisites

Step 1) Constituting the team of the decision makers (DMS): The team is developed to identify criteria to evaluate distributors. A brainstorming session or meeting can be held in order to determine the required criteria for distribution according to the product and supply chain of the manufacturing company.

Step 2) Evaluation of distributors: The team determines the grading scale to rate each distributor according to the defined criteria.

Phase 2. Training

To deal with the drawbacks of the fuzzy ART algorithm, it has been proposed to train the neural network. During training, the neural network categorizes natural patterns of data into groups with similar features, when it is confronted by a new input it produces a response that indicates which category the pattern belongs to. The training phase of the fuzzy ART works as follows. Given a list of input patterns, designated as $I^{1}, I^{2}, \ldots, I^{p}$, we want to train the fuzzy ART to categorize these input patterns into different categories. Obviously, patterns that are similar to each other are expected to be clustered in the same category by the fuzzy ART. In order to achieve the mentioned goal, the training 
list is presented to the fuzzy ART architecture. The training list is presented as many times as it is necessary for the fuzzy ART to cluster the input patterns. The clustering task is considered accomplished, if the weights in the fuzzy ART architecture do not change during list presentation. The above training scenario is called off-line training. The step-by-step implementation of training is given in the Appendix of this paper.

Phase 3. Categorizing distributors using the fuzzy ART

In this phase, the steps of the fuzzy ART algorithm are described below.

Step 1) Initialize the network: In this step, the initial parameters should be determined by the team of the DMs. Parameters for the fuzzy ART algorithm are vigilance threshold $(\rho)$ and choice $(\alpha) . \rho$ isresponsible for the number of categories, where $\rho \in[0,1]$. If $\rho$ is small, the result is inclined to a rough categorization. On the other hand, if $\rho$ is chosen to be close to 1 , many finely divided categories are formed and similarity in each category is much higher and choice parameter $\alpha$ is effective in category selection. These parameters are determined based on the type of the problem.

The initial weights for all $i$ and $j$ are taken from the trained network. Also, the number of category is set to the categories in the trained neural network.

where $i(i=1,2, \ldots, m)$ is the selected distributor and $j(j=1,2, \ldots, n)$ is the criteria number.

Step 2. Normalization of inputs: Using Eq. 1, each input is first normalized.

$$
N I_{i, j}=\frac{I_{i, j}-\min _{j}}{\max _{j}-\min _{j}}
$$

Step 3. Complement coding: Complement coding transforms an $M$-dimensional feature vector I into a $2 M$-dimensional system input vector. A complement-coded system input represents both the degree to which a feature $i$ is present $\left(a_{i}\right)$ and the degree to which that feature is absent $\left(1-a_{i}\right)$.

$$
A_{c}=\left(a_{1}, a_{2}, \ldots, a_{p}, 1-a_{1}, \ldots, 1-a_{p}\right)
$$

Step 4. Presentation of the input vector NI to the network

Step 5. Computation of choice function: Compute the choice function for each existed output node. The choice function is defined by:

$T_{i, s}=\frac{\sum_{j=1}^{n}\left(N I_{i, j} \wedge W_{i, j, s}\right)}{\alpha+\sum_{j=1}^{n} W_{i, j, s}}$

where $\wedge$ is fuzzy 'AND' operator and work as minimum function (i.e., $x \wedge y=\min (x, y)$ ).

Step 6. Selection of maximum choice function value: The maximum choice function value is selected by:

$T^{*}=\max \left\{T_{i, s} ; S=1,2, \ldots k\right\}$

Step 7. Resonance test: The resonance test defines the appropriate category for the input. Computation of matching function is computed by:

$$
M_{i, s}\left(T^{*}\right)=\frac{\sum_{j=1}^{n}\left(N I_{i, j} \wedge W_{i, j, s}\right)}{\sum_{j=1}^{n} N I_{i, j}}
$$

Step 8. Similarity Check: If $M_{i, s}>\rho$, then $T_{i, s}$ is passing the test. Thus, the $i$-th distributor is added to the existing category $C_{s}$, and go to Step 10 . If $M_{i, s}<\rho$ then, $T_{i, s}$ is not passing the test, then go to Step 9.

Step 9. Resetting: Set the choice function value as $T_{i, s}=-1$, and then go back to Step 5. Control the next highest $T_{i, s}$ value. In this way, the matching test continues for all of the $T_{i, s}$ values. If none of $T_{i, s}$ passes the test, a new category is created for the existing input. Thus, the $i$-th distributor is added to the new category $C_{s+1}$. Then, go to Step 4 and compute $T_{i, s}$ for the next input. 
Step 10. Repeat: The algorithm continues with the next input at Step 4. The algorithm ends by allocating all inputs to the categories.

Phase 4. Prioritization

Step 1. Prioritization of categories: The categorized distributors should be prioritized in this step. The arithmetic mean of the input values in each class is used for prioritization. The priorities are determined by this measure and further actions will be done according to this ranking by the team.

\section{Numerical example}

This section considers a numerical example to illustrate the applications of the proposed algorithm. There are 40 distributors and the objective is to categorize them according to 27 criteria. Distributors are scored for every criterion by using the scoring scale shown in Table 2.

\section{Table 2}

Scoring scale

\begin{tabular}{lccccc}
\hline Grading & 5 & 4 & 3 & 2 & 1 \\
\hline Status & Definitely satisfactory & Satisfactory & Average & Unsatisfactory & Definitely unsatisfactory \\
\hline
\end{tabular}

After that, the decision matrix is constituted (see Table 3). As seen from Table 4, similar distributors are clustered in the same category. It is worth to note that before implementing the fuzzy ART algorithm, it has been trained with random numbers. Considering case studies in real cases, data of the previous years can be considered to train the algorithm. According to the priority measure, the most preferred distributors are in the category A. Similarly, other categories are defined and the DM can select the distributors according to their relative priority. Obviously, distributors with the highest priority are more appropriate to be considered. According to the solution of this method, 5, 11, 14 and 10 distributors are realized as most preferred, preferred, recommended and not recommended for the company, respectively. The number of categories depends on the vigilance threshold. If $\rho$ is small, the result is inclined to a coarse categorization. On the other hand, if $\rho$ is chosen to be close to 1 , many finely divided categories are formed. Fig. 3 shows the number of categories considering different vigilance threshold. This enables the DM to determine the number of category according to the sensitivity and complexity of the problem.

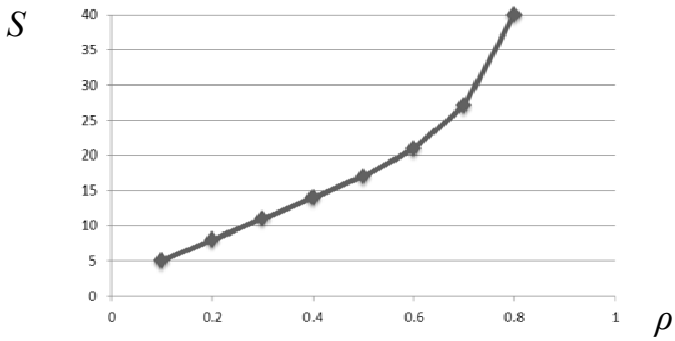

Table 4

Fig. 3. Effect of vigilance threshold $(\rho)$ on number of categories $(\mathrm{S})$

Category membership of the distributors

\begin{tabular}{llll}
\hline Category label & Distributor & Category definition & Priority measure \\
\hline Category A & D23-D34-D35-D36-D38 & Most preferred & 3.126 \\
Category B & D3-D5-D8-D9-D10-D13-D22-D24-D30-D37-D39 & Preferred & 3.091 \\
Category C & D7-D12-D18-D19-D20-D40 & Recommended & 3.019 \\
\hline Category D & D1-D11-D15-D17-D21-D27-D32-D33 & Recommended & 3.019 \\
Category E & D14-D16-D28-D29-D31 & Not recommended & 2.934 \\
Category F & D2 & Not recommended & 2.889 \\
Category G & D4-D6-D25-D26 & Not recommended & 2.889 \\
\hline
\end{tabular}


Table 3

Alternative distributors and their grade

\begin{tabular}{|c|c|c|c|c|c|c|c|c|c|c|c|c|c|c|c|c|c|c|c|c|c|c|c|c|c|c|c|}
\hline \multirow[b]{3}{*}{ Distributor } & \multicolumn{27}{|c|}{ Criteria } \\
\hline & 1 & 2 & 3 & 4 & 5 & 6 & 7 & 8 & 9 & 10 & 11 & 12 & 13 & 14 & 15 & 16 & 17 & 18 & 19 & 20 & 21 & 22 & 23 & 24 & 25 & 26 & 27 \\
\hline & \multicolumn{27}{|c|}{ Grades } \\
\hline D1 & 3 & 5 & 5 & 1 & 5 & 1 & 1 & 5 & 2 & 5 & 2 & 5 & 3 & 2 & 4 & 4 & 2 & 1 & 5 & 4 & 2 & 2 & 5 & 4 & 3 & 4 & 5 \\
\hline D2 & 2 & 2 & 1 & 3 & 5 & 1 & 4 & 1 & 1 & 5 & 2 & 1 & 4 & 3 & 4 & 1 & 1 & 5 & 5 & 2 & 1 & 5 & 2 & 3 & 5 & 4 & 5 \\
\hline D3 & 1 & 3 & 2 & 1 & 3 & 1 & 2 & 3 & 3 & 5 & 3 & 2 & 3 & 3 & 1 & 2 & 4 & 2 & 3 & 5 & 4 & 3 & 5 & 2 & 3 & 2 & 4 \\
\hline D4 & 3 & 2 & 1 & 4 & 4 & 2 & 1 & 5 & 1 & 1 & 3 & 3 & 5 & 2 & 4 & 1 & 5 & 1 & 3 & 3 & 3 & 1 & 5 & 5 & 4 & 5 & 3 \\
\hline D5 & 1 & 4 & 2 & 2 & 5 & 2 & 5 & 2 & 3 & 4 & 1 & 1 & 3 & 5 & 5 & 5 & 5 & 3 & 1 & 4 & 3 & 3 & 3 & 3 & 4 & 5 & 5 \\
\hline D6 & 1 & 5 & 3 & 1 & 2 & 5 & 5 & 3 & 3 & 1 & 3 & 1 & 5 & 3 & 1 & 3 & 5 & 5 & 5 & 4 & 2 & 2 & 1 & 1 & 4 & 5 & 5 \\
\hline D7 & 5 & 2 & 1 & 4 & 3 & 4 & 3 & 5 & 4 & 2 & 1 & 5 & 3 & 1 & 3 & 2 & 3 & 3 & 4 & 2 & 4 & 3 & 2 & 4 & 5 & 3 & 2 \\
\hline D8 & 5 & 1 & 1 & 5 & 5 & 5 & 3 & 4 & 3 & 4 & 5 & 1 & 5 & 2 & 5 & 2 & 2 & 1 & 5 & 3 & 2 & 2 & 5 & 4 & 2 & 2 & 5 \\
\hline D9 & 3 & 1 & 3 & 5 & 5 & 2 & 5 & 2 & 3 & 4 & 5 & 3 & 3 & 4 & 1 & 4 & 4 & 1 & 5 & 4 & 1 & 1 & 2 & 5 & 2 & 4 & 2 \\
\hline D10 & 5 & 3 & 5 & 2 & 1 & 1 & 3 & 3 & 4 & 4 & 4 & 3 & 5 & 3 & 3 & 3 & 3 & 4 & 2 & 4 & 5 & 1 & 5 & 2 & 3 & 5 & 3 \\
\hline D11 & 3 & 3 & 5 & 5 & 5 & 3 & 3 & 2 & 1 & 1 & 3 & 2 & 2 & 4 & 2 & 2 & 3 & 3 & 2 & 4 & 2 & 3 & 3 & 2 & 3 & 1 & 3 \\
\hline D12 & 1 & 2 & 1 & 4 & 3 & 1 & 5 & 5 & 2 & 2 & 1 & 3 & 4 & 1 & 3 & 2 & 2 & 2 & 4 & 3 & 5 & 2 & 1 & 5 & 5 & 1 & 4 \\
\hline D13 & 5 & 1 & 2 & 5 & 3 & 2 & 3 & 3 & 1 & 1 & 1 & 1 & 5 & 4 & 5 & 1 & 3 & 1 & 5 & 5 & 1 & 2 & 5 & 2 & 4 & 3 & 4 \\
\hline D14 & 3 & 5 & 2 & 5 & 3 & 3 & 2 & 4 & 4 & 1 & 4 & 3 & 3 & 2 & 3 & 2 & 2 & 3 & 2 & 3 & 5 & 3 & 2 & 5 & 5 & 3 & 3 \\
\hline D15 & 3 & 5 & 5 & 2 & 1 & 4 & 3 & 3 & 1 & 5 & 2 & 4 & 1 & 2 & 2 & 5 & 4 & 1 & 1 & 2 & 1 & 5 & 1 & 1 & 1 & 1 & 2 \\
\hline D16 & 5 & 4 & 3 & 3 & 3 & 3 & 3 & 3 & 5 & 4 & 2 & 3 & 5 & 5 & 4 & 2 & 3 & 5 & 2 & 5 & 5 & 1 & 4 & 4 & 1 & 5 & 2 \\
\hline D17 & 2 & 4 & 4 & 4 & 1 & 3 & 5 & 3 & 4 & 5 & 3 & 5 & 3 & 2 & 4 & 5 & 3 & 2 & 2 & 2 & 1 & 4 & 4 & 2 & 2 & 3 & 3 \\
\hline D18 & 5 & 5 & 4 & 3 & 5 & 2 & 4 & 3 & 2 & 3 & 1 & 2 & 1 & 1 & 3 & 4 & 4 & 3 & 2 & 4 & 5 & 5 & 2 & 4 & 5 & 2 & 2 \\
\hline D19 & 4 & 3 & 3 & 3 & 4 & 1 & 3 & 5 & 3 & 5 & 2 & 4 & 1 & 2 & 5 & 1 & 1 & 3 & 5 & 5 & 5 & 1 & 4 & 5 & 4 & 4 & 4 \\
\hline $\mathrm{D} 20$ & 5 & 5 & 1 & 2 & 2 & 5 & 5 & 5 & 3 & 5 & 2 & 2 & 1 & 2 & 1 & 2 & 4 & 5 & 2 & 1 & 2 & 5 & 5 & 3 & 3 & 2 & 2 \\
\hline D21 & 1 & 2 & 4 & 1 & 3 & 5 & 3 & 5 & 5 & 3 & 3 & 5 & 5 & 2 & 2 & 3 & 1 & 4 & 5 & 3 & 2 & 2 & 5 & 1 & 3 & 2 & 4 \\
\hline D22 & 3 & 1 & 4 & 3 & 2 & 2 & 4 & 2 & 4 & 5 & 3 & 1 & 5 & 4 & 2 & 5 & 1 & 3 & 5 & 2 & 5 & 1 & 5 & 2 & 4 & 5 & 4 \\
\hline $\mathrm{D} 23$ & 5 & 4 & 5 & 1 & 5 & 1 & 3 & 1 & 2 & 2 & 4 & 3 & 1 & 3 & 4 & 4 & 5 & 5 & 1 & 4 & 1 & 4 & 3 & 5 & 5 & 2 & 5 \\
\hline D24 & 1 & 3 & 3 & 5 & 3 & 4 & 3 & 4 & 4 & 2 & 1 & 2 & 5 & 5 & 3 & 5 & 4 & 1 & 3 & 3 & 1 & 4 & 4 & 5 & 2 & 2 & 4 \\
\hline D25 & 4 & 2 & 2 & 3 & 1 & 4 & 3 & 5 & 5 & 4 & 1 & 1 & 1 & 1 & 3 & 2 & 1 & 2 & 2 & 3 & 3 & 2 & 5 & 1 & 1 & 1 & 5 \\
\hline D26 & 3 & 3 & 2 & 4 & 3 & 1 & 5 & 2 & 4 & 1 & 4 & 4 & 5 & 4 & 1 & 1 & 5 & 2 & 5 & 5 & 2 & 1 & 1 & 2 & 3 & 5 & 2 \\
\hline D27 & 1 & 5 & 5 & 5 & 5 & 5 & 4 & 4 & 2 & 5 & 3 & 1 & 1 & 3 & 4 & 4 & 5 & 1 & 2 & 4 & 5 & 1 & 1 & 2 & 1 & 5 & 5 \\
\hline D28 & 5 & 3 & 4 & 1 & 5 & 2 & 5 & 3 & 4 & 3 & 4 & 1 & 3 & 3 & 1 & 3 & 2 & 5 & 5 & 3 & 2 & 4 & 3 & 4 & 2 & 2 & 1 \\
\hline D29 & 1 & 2 & 4 & 1 & 5 & 1 & 1 & 5 & 5 & 3 & 3 & 5 & 1 & 3 & 2 & 1 & 2 & 4 & 4 & 2 & 2 & 1 & 4 & 1 & 4 & 3 & 3 \\
\hline D30 & 3 & 1 & 2 & 4 & 2 & 5 & 2 & 4 & 4 & 5 & 1 & 2 & 5 & 5 & 4 & 3 & 1 & 1 & 5 & 4 & 2 & 4 & 4 & 2 & 2 & 2 & 3 \\
\hline D31 & 3 & 1 & 1 & 2 & 1 & 1 & 1 & 3 & 3 & 3 & 2 & 3 & 5 & 1 & 3 & 3 & 4 & 2 & 1 & 1 & 5 & 1 & 3 & 1 & 1 & 4 & 2 \\
\hline D32 & 1 & 3 & 3 & 1 & 5 & 4 & 3 & 4 & 1 & 4 & 5 & 5 & 4 & 4 & 3 & 4 & 5 & 2 & 3 & 1 & 5 & 3 & 1 & 2 & 1 & 3 & 1 \\
\hline D33 & 4 & 4 & 4 & 2 & 4 & 4 & 4 & 2 & 5 & 1 & 4 & 1 & 3 & 4 & 4 & 1 & 2 & 5 & 1 & 1 & 4 & 1 & 4 & 1 & 2 & 5 & 3 \\
\hline D34 & 4 & 4 & 5 & 5 & 4 & 5 & 2 & 2 & 4 & 5 & 5 & 5 & 5 & 5 & 5 & 5 & 2 & 5 & 5 & 2 & 4 & 4 & 2 & 5 & 3 & 5 & 4 \\
\hline D35 & 1 & 2 & 2 & 5 & 4 & 5 & 2 & 1 & 1 & 3 & 4 & 3 & 5 & 4 & 4 & 5 & 2 & 3 & 2 & 2 & 4 & 2 & 3 & 1 & 2 & 5 & 2 \\
\hline D36 & 4 & 2 & 1 & 4 & 1 & 5 & 2 & 1 & 1 & 4 & 4 & 5 & 2 & 4 & 1 & 5 & 2 & 1 & 4 & 2 & 1 & 4 & 2 & 3 & 3 & 3 & 5 \\
\hline D37 & 5 & 2 & 4 & 1 & 2 & 3 & 3 & 5 & 3 & 3 & 4 & 3 & 3 & 5 & 1 & 1 & 2 & 1 & 5 & 4 & 5 & 3 & 5 & 3 & 3 & 1 & 5 \\
\hline D38 & 3 & 2 & 3 & 4 & 2 & 1 & 1 & 2 & 5 & 1 & 1 & 2 & 5 & 1 & 2 & 1 & 2 & 3 & 2 & 4 & 3 & 4 & 2 & 2 & 2 & 3 & 5 \\
\hline D39 & 3 & 2 & 1 & 1 & 2 & 4 & 3 & 2 & 5 & 4 & 4 & 5 & 3 & 3 & 1 & 5 & 1 & 1 & 3 & 4 & 3 & 2 & 1 & 2 & 4 & 3 & 2 \\
\hline D40 & 3 & 1 & 2 & 3 & 2 & 5 & 4 & 2 & 3 & 2 & 4 & 5 & 2 & 1 & 5 & 1 & 5 & 2 & 1 & 2 & 4 & 4 & 1 & 2 & 3 & 2 & 3 \\
\hline
\end{tabular}

\section{Conclusions}

In this paper, distributor selection and categorization have been conducted through the fuzzy Adaptive Resonance Theory (ART) algorithm. In the first step, criteria have been defined by the decision makers (DMs), and then these DMs have used a grading scale to rate each distributor regarding these criteria. Furthermore, the fuzzy ART algorithm has been utilized to cluster the distributors with similar features. The proposed approach has enhanced the clustering algorithm proposed by Aydın Keskin et al. (2006) for supplier selection. Then, the numerical example has been conducted to show the effectiveness of our proposed approach. Partner selection problems have been solved by numerous methods. In the context of supplier selection and evaluation, MODM techniques (e.g., goal programming) and MCDM techniques (e.g., AHP, ANP and TOPSIS methods) have been used widely along with mathematical programming methods. On the other hand, in the context of distributor selection and evaluation, few studies could be found in the literature. These studies have utilized MCDM and MODM techniques, rough set theory and artificial intelligence (AI) to deal with this problem.

In reality, when the complexity and ambiguity of information is high, AI methods are better than traditional methods, because they are designed to act like human judgment. In addition, they can learn from the past data. Therefore, the decision maker (DM) should only provide the information needed for the system. The most important contribution of the proposed method was the ability of its clustering for the distributor selection and evaluation problems. The distributors are clustered according to their similarity degrees between them. The fuzzy ART not only determines the best 
distributors, but also clusters all distributors. This procedure has been very effective in partner selection and evaluation problems. In addition, the drawbacks of the algorithm have been mitigated by training the neural network. The algorithm has been adaptive and has easily applied to firms and companies. This method has been very flexible, and a number of categories have been different by changing the vigilance parameter. The algorithm has been especially good for the large-sized data and its simplicity made it applicable.

\section{Appendix A: The ART training algorithm}

The fuzzy ART operates over all of the committed nodes along with a single uncommitted node. Each committed node of index $j$ has a vector $W_{j}=\left(W_{j 1}, W_{j 2}, \ldots, W_{j 2 . p}\right)$ of adaptive weights, which represents the coded template. The number of committed nodes $J(j=1, \ldots, J)$ is arbitrary, while the dimension of vector $w$ is $2 p$. Let $x$ be a $P$-dimensional input vector $x=\left(x_{1}, \ldots, x_{p}\right)$, where each component $x_{p}$ ranges in $[0,1]$. The matching algorithm of the fuzzy ART is as follows.

Step 1. Initialization: During training, initialize the number of committed nodes to $J=0$. Then, set a choice parameter $\alpha \in[0, \infty]$ (a small value is used in this work, say $\alpha=10^{-6}$ ), and a vigilance parameter $\rho \in[0,1]$.

Step 2. Complement coding: Expand each new input $x$ into the $2 P$-dimensional vector $x$ defined by:

$x_{c}=\left(x_{1}, \ldots, x_{p}, 1-x_{1}, \ldots, 1-x_{p}\right)$.

Step 3. Category choice: For each committed node of index $j=1, \ldots, J$, compute the bottom-up input $T_{j}$ as follows,

$T_{j}=\frac{\sum_{j=1}^{n}\left(x_{c} \wedge W_{j}\right)}{\alpha+\sum_{j=1}^{n} W_{j}}$.

Choose the committed node in F2 that receives the maximum bottom-up input. Assume this node has index $j^{*} T_{j^{*}}=\max \left\{T_{j} ; j=1,2, \ldots J\right\}$. If more than one $T_{j^{*}}$ is maximal, choose the category with the smallest $j$ index. Two cases can be distinguished.

- If there are no categories for classifying the current input, release an alarm. During training, select the uncommitted node by setting $J=J+1, j^{*}=J$ and $W_{j^{*}}=x_{c}$. Introduce a new uncommitted node in layer F2, and then go to the beginning of Step 2.

- Otherwise, go to Step 4.

Step 4. Resonance or reset: Check to see whether node $j^{*}$ satisfies the following vigilance criterion.

$$
\frac{\sum_{j=1}^{n}\left(x_{c} \wedge W_{j^{*}}\right)}{\sum_{j=1}^{n} x_{c}} \geq \rho
$$

Two cases can be distinguished.

- If the vigilance criterion is satisfied, the current input is classified in the category of index $j^{*}$ (no alarm is released). During training, update the weight vector $W_{j^{*}}^{\text {new }}=\min \left(x_{c}, W_{j^{*}}^{\text {old }}\right)$ (i.e., fast learning), and then go to the beginning of Step 2.

- Otherwise, exclude the node $j^{*}$ by setting the choice function $T_{j^{*}}=-1$ for the duration of the input presentations to prevent its persistent selection during the search, and then go to the beginning of Step 3.

\section{References}

Aydın Keskin, G., Ilhan, S. \& Ozkan, C. (2010). The Fuzzy ART algorithm: A categorization method for supplier evaluation and selection, Expert Systems with Applications, 37, 1235-1240. 
Aydın Keskin, \& Ozkan, C. (2009). A new evaluation method for FMEA: Fuzzy ART algorithm. Quality and Reliability Engineering International, 25, 647-661.

Bhattacharya, A., Geraghty, J. \& Young, P. (2010). Supplier selection paradigm: An integrated hierarchical QFD methodology under multiple-criteria environment. Applied Soft Computing, 10(4), 1013-1027.

Fassnacht, M. \& Koese, I. (2006). Quality of electronic services: conceptualizing and testing a hierarchical model. Journal of Service Research, 9 (1), 19-37.

Hugos, M. (2006). Essentials of supply chain management, second edition. John Wiley \& Sons, Inc.

Kuo, M.S., \& Liang, G.S. (2011).Combining VIKOR with GRA techniques to evaluate service quality of air ports under fuzzy environment. Expert Systems with Applications, 38, 1304-1312.

Lin, J.S.C. \& Chen, C.R. (2008). Determinants of manufacturers' selection ofdistributors. Supply Chain Management: An International Journal, 13(5), 356-365.

Lopes, M.L.M., Minussi, C.R. \& Lotufo, A.D.P. (2005). Electric load forecasting using a Fuzzy ART \& ARTMAP neural network. Applied Soft Computing, 5, 235-244.

Mousavi, S.M., Jolai, F. \& Tavakkoli-Moghaddam, R. (2011a). A new fuzzy stochastic multiattribute group decision-making approach for selection problems. Group Decision and Negotiation, to appear in 2011, DOI: 10.1007/s10726-011-9259-1.

Mousavi, S.M., Tavakkoli-Moghaddam, R., Heydar, M. \& Ebrahimnejad, S. (2011b). Multi-criteria decision-making for plant location selection: an integrated Delphi-AHP-PROMETHEE methodology. Arabian Journal for Science and Engineering (AJSE: B-Engineering), accepted for publication, to appear in 2011.

Narasimhan, R., Talluri, S. \& Mahapatra, S.K. (2006). Multiproduct, multicriteria model for supplier selection with product life-cycle considerations. Decision Sciences, 37(4), 577-603.

Pacella, M., Semeraro, Q. \& Anglani, A. (2004). Manufacturing quality control by means of a Fuzzy ART network trained on natural process data. Artificial Intelligence, 17, (83-96).

Pacella, M. \& Semeraro, Q. (2011). Monitoring roundness profiles based on an unsupervised neural network algorithm. Computers \& Industrial Engineering, 60(4), 677-689.

Pandian, R.S. \& Mahapatra, S.S. (2009).Manufacturing cell formation with production data using neural networks.Computers \& Industrial Engineering, 56, 1340-1347.

Punniyamoorthy, M., Mathiyalagan, P. \& Parthiban, P. (2011). A strategic model using structural equation modeling and fuzzy logic in supplier selection. Expert Systems with Applications, 38(1), $458-474$.

Razmi, J., Songhori, M.J. \& Khakbaz, M.H. (2009). An integrated fuzzy group decision making/fuzzy linear programming (FGDMLP) framework for supplier evaluation and order allocation. International Journal of Advanced Manufacturing Technology, 43(6-7), 590-607.

Sharma, D., Sahay, B.S. \& Sachan, A. (2004). Modeling distributor performance index using system dynamics approach. Asia Pacific Journal of Marketing and Logistics, 16, 37-67.

Talluri, S., \& Narasimhan, R. (2005).A note on a methodology for supply base optimization. IEEE Transactions on Engineering Management, 52(1), 130-139.

Vanteddu, G., Chinnam, R.B., \& Gushikin, O. (2010). Supply chain focus dependent supplier selection problem. International Journal of Production Economics, 129(1), 204-216.

Wang, J.W., Cheng, C.H. \& Huang, K.C. (2009). Fuzzy hierarchical TOPSIS for supplier selection. Applied Soft Computing, 9(1), 377-386.

Wang, L.,\& Kess, P. (2006).Partnering motives and partner selection, case study of finish distributor relationships in china. International Journal of Physical Distribution and Logistics Management, $36(6), 466-478$.

Zou, Z., Tseng, T.L., Sohn, H., Song, G. \& Gutierrez, R. (2011). A rough set based approach to distributor selection in supply chain management. Expert Systems with Applications, 38, 106-115. 